\title{
BMC Biotechnology reviewer acknowledgement 2014
}

Dafne Solera

\section{Contributing reviewers}

The editors of BMC Biotechnology would like to thank all our reviewers who have contributed to the journal in Volume 14 (2014).

D. Wade Abbott

Canada

Karolina Aberg

USA

Gabriela Almeida

Portugal

Sara Amancio

Portugal

Thomas Anchordoquy

USA

Denis Archambault

Canada

Hideyuki Arimitsu

Japan

Aglaia Athanassiadou

Greece

Tamas Atlasz

Hungary

Brian Austin

USA

Eduard Ayuso

France

Matteo Ballottari

Italy

Jo Ann Banks

USA
Priya Baraniak
USA

Susanne Barth

Ireland

Kristin Baumann

Austria

Kyoungwhan Back

South Korea

Robert Belshaw

UK

Per Berglund

Sweden

Frank Bernhard

Germany

Maurizio Bettiga

Sweden

Andriy Bilichak

Canada

Lars Mathias Blank

Germany

Murray Boase

New Zealand

Mariela Bollati-Fogolin

Uruguay

Massimiliano Borgogna

Italy

\author{
Abhijeet Borole \\ USA \\ Berrin Bozan \\ Turkey \\ Mike Brisco \\ Australia \\ Jochen Büchs \\ Germany \\ Jan Buer \\ Germany \\ Pradeep Burma \\ India \\ John Burnett \\ USA \\ Azeem Butt \\ Pakistan \\ Cecília Calado \\ Portugal \\ Maria Cammareri \\ Italy \\ Lixiang Cao \\ China \\ Yongchang Cao \\ China \\ Sheng-Hao Chao \\ Singapore
}

Correspondence: Dafne.solera@biomedcentral.com

BioMed Central, Floor 6, 236 Gray's Inn Road, London WC1X 8HB, UK 


Bhushan Chaudhari
India
Guo-Qiang Chen
China
Sanfeng Chen
China
Shyan-Song Chiou
Taiwan
Raymond Chollet
USA
Yi Chu
USA
Dennis Claessen
Netherlands
Patrick Coates
Australia
Michele Colonna
Italy
Gregory Cote
USA

Rosymar Coutinho De Lucas Brazil

Don Cowan South

Africa

Monika Cserjan

Austria

Richard Cummings

USA

Rosa M. Cusido

Spain

Andre Damasio

Brazil

Henri Darmency

France

Kasturi Dasgupta

USA

Shiladitya Dassarma

USA

Lisandra De Castro Bras USA

Ario De Marco

Slovenia

Elena Deineko

Russian Federation

\begin{tabular}{ll} 
Zujun Deng & Melissa Fullwood \\
China & Singapore \\
Ratmir Derda & Bruria Funkenstein \\
Canada & Israel \\
Sheng-Li Ding & Sushama Gaikwad \\
USA & India \\
Yu Ding & Junping Gao \\
China & China \\
Ron Dirks & Manuel Garrido \\
Netherlands & Portugal \\
Philip Dix & Brigitte Gasser \\
Ireland & Austria \\
Jozsef Dobo & Elena Geiser \\
Hungary & Germany \\
Christian Dölle & Richard Gemeinhart \\
Norway & USA \\
Jinhua Dong & \\
\hline
\end{tabular}

Andrea Germer

Germany

Ellen Goldman

USA

Sailaja Gopalakrishnanchettiar Sivakamiammal

USA

Billie Gould

Canada

Sibylle Grad

Switzerland

Mario Graos

Portugal

Rita Graze

USA

Teresa Cristina Grisi

Brazil

Man Bock Gu

South Korea

USA

Paola Foladori

Italy

Diogo Fonseca-Pereira

Portugal

Bedon Frank

Australia

Maria Frantzi

Germany
Santiago Gutierrez

Spain

Matthias Hackl

Austria

Donny Hanjaya-Putra

USA

Michiel Harmsen
Netherlands 


\begin{tabular}{|c|c|c|}
\hline $\begin{array}{l}\text { Colin Harwood } \\
\text { UK }\end{array}$ & $\begin{array}{l}\text { Su Jianguang } \\
\text { China }\end{array}$ & $\begin{array}{l}\text { Harald Kolmar } \\
\text { Germany }\end{array}$ \\
\hline $\begin{array}{l}\text { Rajni Hatti Kaul } \\
\text { Sweden }\end{array}$ & $\begin{array}{l}\text { Jonathan Jones } \\
\text { UK }\end{array}$ & $\begin{array}{l}\text { Bhupendra Koul } \\
\text { India }\end{array}$ \\
\hline $\begin{array}{l}\text { Dimitris Hatzinikolaou } \\
\text { Greece }\end{array}$ & $\begin{array}{l}\text { Leif J Jonsson } \\
\text { Sweden }\end{array}$ & $\begin{array}{l}\text { Andrew Kralicek } \\
\text { New Zealand }\end{array}$ \\
\hline $\begin{array}{l}\text { Klaas Jan Hellingwerf } \\
\text { Netherlands }\end{array}$ & $\begin{array}{l}\text { Franziska Jönsson } \\
\text { Germany }\end{array}$ & $\begin{array}{l}\text { Florian Krammer } \\
\text { USA }\end{array}$ \\
\hline $\begin{array}{l}\text { Taira Hidaka } \\
\text { Japan }\end{array}$ & $\begin{array}{l}\text { Christian Jungreuthmayer } \\
\text { Austria }\end{array}$ & $\begin{array}{l}\text { Harald Kranz } \\
\text { Germany }\end{array}$ \\
\hline $\begin{array}{l}\text { Maria Eugenia Hidalgo Lara } \\
\text { Mexico }\end{array}$ & $\begin{array}{l}\text { Eszter Kapusi } \\
\text { Austria }\end{array}$ & $\begin{array}{l}\text { Anja Kuenz } \\
\text { Germany }\end{array}$ \\
\hline $\begin{array}{l}\text { Thomas Higgins } \\
\text { Australia }\end{array}$ & $\begin{array}{l}\text { Levente Karaffa } \\
\text { Hungary }\end{array}$ & $\begin{array}{l}\text { Wilfried Kues } \\
\text { Germany }\end{array}$ \\
\hline $\begin{array}{l}\text { Nobutaka Hirano } \\
\text { Japan }\end{array}$ & $\begin{array}{l}\text { Georgios Kararigas } \\
\text { Germany }\end{array}$ & $\begin{array}{l}\text { Peter Kuhnert } \\
\text { Switzerland }\end{array}$ \\
\hline $\begin{array}{l}\text { Corinne Hoesli } \\
\text { Canada }\end{array}$ & $\begin{array}{l}\text { Tadas Kasputis } \\
\text { USA }\end{array}$ & $\begin{array}{l}\text { Prakash Kumar } \\
\text { Singapore }\end{array}$ \\
\hline $\begin{array}{l}\text { Dirk Hoffmeister } \\
\text { Germany }\end{array}$ & $\begin{array}{l}\text { Tatsuya Kato } \\
\text { Japan }\end{array}$ & $\begin{array}{l}\text { Jochen Kumlehn } \\
\text { Germany }\end{array}$ \\
\hline $\begin{array}{l}\text { Mads Vilhelm Hollegaard } \\
\text { Denmark }\end{array}$ & $\begin{array}{l}\text { Rajinder Kaul } \\
\text { USA }\end{array}$ & $\begin{array}{l}\text { Masahiro Kurakake } \\
\text { Japan }\end{array}$ \\
\hline $\begin{array}{l}\text { Hyo Jeong Hong } \\
\text { South Korea }\end{array}$ & $\begin{array}{l}\text { Yoshinori Kawabe } \\
\text { Japan }\end{array}$ & $\begin{array}{l}\text { Sunil Kurian } \\
\text { USA }\end{array}$ \\
\hline $\begin{array}{l}\text { Danny Hooftman } \\
\text { UK }\end{array}$ & $\begin{array}{l}\text { Arvind Kayastha } \\
\text { India }\end{array}$ & $\begin{array}{l}\text { Sang-Soo Kwak } \\
\text { South Korea }\end{array}$ \\
\hline $\begin{array}{l}\text { Chienjin Huang } \\
\text { Taiwan }\end{array}$ & $\begin{array}{l}\text { Nawazi Khan } \\
\text { USA }\end{array}$ & $\begin{array}{l}\text { Ying Lai } \\
\text { USA }\end{array}$ \\
\hline $\begin{array}{l}\text { Zhaohui Huang } \\
\text { China }\end{array}$ & $\begin{array}{l}\text { Muhammad Azmat Ullah Khan } \\
\text { USA }\end{array}$ & $\begin{array}{l}\text { Marcelo Lancellotti } \\
\text { Brazil }\end{array}$ \\
\hline $\begin{array}{l}\text { Matt Hutchings } \\
\text { UK }\end{array}$ & $\begin{array}{l}\text { Jin Kim } \\
\text { USA }\end{array}$ & $\begin{array}{l}\text { Ivan Lavandera } \\
\text { Spain }\end{array}$ \\
\hline $\begin{array}{l}\text { Ee Taek Hwang } \\
\text { South Korea }\end{array}$ & $\begin{array}{l}\text { Yeon-Gu Kim } \\
\text { South Korea }\end{array}$ & $\begin{array}{l}\text { Gerald Leblanc } \\
\text { USA }\end{array}$ \\
\hline $\begin{array}{l}\text { Hafiz Muhammad Nasir Iqbal } \\
\text { Pakistan }\end{array}$ & $\begin{array}{l}\text { Nobutada Kimura } \\
\text { Japan }\end{array}$ & $\begin{array}{l}\text { Bruno Lefebvre } \\
\text { France }\end{array}$ \\
\hline $\begin{array}{l}\text { Tomohiro Ishikawa } \\
\text { Japan }\end{array}$ & $\begin{array}{l}\text { David Kirk } \\
\text { USA }\end{array}$ & $\begin{array}{l}\text { Jean-Luc Lenormand } \\
\text { France }\end{array}$ \\
\hline $\begin{array}{l}\text { Medicharla V Jagannadham } \\
\text { India }\end{array}$ & $\begin{array}{l}\text { Hiroshi Kitagaki } \\
\text { Japan }\end{array}$ & $\begin{array}{l}\text { Thierry Leroy } \\
\text { France }\end{array}$ \\
\hline $\begin{array}{l}\text { Sharmili Jagtap } \\
\text { India }\end{array}$ & $\begin{array}{l}\text { Dieter Klemm } \\
\text { Germany }\end{array}$ & $\begin{array}{l}\text { Randolph Lewis } \\
\text { USA }\end{array}$ \\
\hline $\begin{array}{l}\text { Grzegorz Janusz } \\
\text { Poland }\end{array}$ & $\begin{array}{l}\text { Heath Klock } \\
\text { USA }\end{array}$ & $\begin{array}{l}\text { Dan Li } \\
\text { China }\end{array}$ \\
\hline
\end{tabular}




\begin{tabular}{|c|c|c|}
\hline $\begin{array}{l}\text { Zhimin Li } \\
\text { China }\end{array}$ & $\begin{array}{l}\text { Ludmila Martinkova } \\
\text { Czech Republic }\end{array}$ & $\begin{array}{l}\text { Neftali Ochoa-Alejo } \\
\text { Mexico }\end{array}$ \\
\hline $\begin{array}{l}\text { Lijia Li } \\
\text { China }\end{array}$ & $\begin{array}{l}\text { Carla Marusic } \\
\text { Italy }\end{array}$ & $\begin{array}{l}\text { Marco Oldiges } \\
\text { Germany }\end{array}$ \\
\hline $\begin{array}{l}\text { Ruifang Li } \\
\text { China }\end{array}$ & $\begin{array}{l}\text { Kazuya Matsumoto } \\
\text { Japan }\end{array}$ & $\begin{array}{l}\text { Berit Olsen Krogh } \\
\text { Denmark }\end{array}$ \\
\hline $\begin{array}{l}\text { Gang Li } \\
\text { China }\end{array}$ & $\begin{array}{l}\text { Katsuhisa Matsuura } \\
\text { Japan }\end{array}$ & $\begin{array}{l}\text { Bradley Olson } \\
\text { USA }\end{array}$ \\
\hline $\begin{array}{l}\text { Marc Libault } \\
\text { USA }\end{array}$ & $\begin{array}{l}\text { Autar Mattoo } \\
\text { USA }\end{array}$ & $\begin{array}{l}\text { Ron Ophir } \\
\text { Israel }\end{array}$ \\
\hline $\begin{array}{l}\text { Fei-Hu Liu } \\
\text { China }\end{array}$ & $\begin{array}{l}\text { Sathiyamoorthy Meiyalaghan } \\
\text { New Zealand }\end{array}$ & $\begin{array}{l}\text { Diego Orzaez } \\
\text { Spain }\end{array}$ \\
\hline $\begin{array}{l}\text { Ji-Kai Liu } \\
\text { China }\end{array}$ & $\begin{array}{l}\text { Wenzhao Meng } \\
\text { USA }\end{array}$ & $\begin{array}{l}\text { Sriram Padmanabhan } \\
\text { India }\end{array}$ \\
\hline $\begin{array}{l}\text { Xiudong Liu } \\
\text { China }\end{array}$ & $\begin{array}{l}\text { Diana Molino } \\
\text { France }\end{array}$ & $\begin{array}{l}\text { Alok Pandey } \\
\text { USA }\end{array}$ \\
\hline $\begin{array}{l}\text { Yiwei Liu } \\
\text { USA }\end{array}$ & $\begin{array}{l}\text { Lluis Montoliu } \\
\text { Spain }\end{array}$ & $\begin{array}{l}\text { Soumya Pandit } \\
\text { India }\end{array}$ \\
\hline $\begin{array}{l}\text { Jian-Zhong Liu } \\
\text { China }\end{array}$ & $\begin{array}{l}\text { Ed Morgan } \\
\text { New Zealand }\end{array}$ & $\begin{array}{l}\text { Hongbo Pang } \\
\text { USA }\end{array}$ \\
\hline $\begin{array}{l}\text { Wusheng Liu } \\
\text { USA }\end{array}$ & $\begin{array}{l}\text { Susann Müller } \\
\text { Germany }\end{array}$ & $\begin{array}{l}\text { Patchareewan Pannangpetch } \\
\text { Thailand }\end{array}$ \\
\hline $\begin{array}{l}\text { Patricia Liwang } \\
\text { USA }\end{array}$ & $\begin{array}{l}\text { Ewen Mullins } \\
\text { Ireland }\end{array}$ & $\begin{array}{l}\text { Francesca Paradisi } \\
\text { Ireland }\end{array}$ \\
\hline $\begin{array}{l}\text { Agnieszka Loboda } \\
\text { Poland }\end{array}$ & $\begin{array}{l}\text { Hideo Nakano } \\
\text { Japan }\end{array}$ & $\begin{array}{l}\text { Ashok Patel } \\
\text { India }\end{array}$ \\
\hline $\begin{array}{l}\text { Wayne Loescher } \\
\text { USA }\end{array}$ & $\begin{array}{l}\text { Abhay Narayan Singh } \\
\text { UK }\end{array}$ & $\begin{array}{l}\text { Stephanie Pearl } \\
\text { USA }\end{array}$ \\
\hline $\begin{array}{l}\text { Catarina Madeira } \\
\text { Portugal }\end{array}$ & $\begin{array}{l}\text { Diana Nascimento } \\
\text { Portugal }\end{array}$ & $\begin{array}{l}\text { Michael Pearson } \\
\text { New Zealand }\end{array}$ \\
\hline $\begin{array}{l}\text { Indu Maiti } \\
\text { USA }\end{array}$ & $\begin{array}{l}\text { Helena Nevalainen } \\
\text { Australia }\end{array}$ & $\begin{array}{l}\text { Nels Pederson } \\
\text { USA }\end{array}$ \\
\hline $\begin{array}{l}\text { Miia Mäkelä } \\
\text { Finland }\end{array}$ & $\begin{array}{l}\text { Pablo Ivan Nikel } \\
\text { Spain }\end{array}$ & $\begin{array}{l}\text { Cristina Peixoto } \\
\text { Portugal }\end{array}$ \\
\hline $\begin{array}{l}\text { Angel Manteca } \\
\text { Spain }\end{array}$ & $\begin{array}{l}\text { Benjamin Manuel Nitsche } \\
\text { Germany }\end{array}$ & $\begin{array}{l}\text { William Plaxton } \\
\text { Canada }\end{array}$ \\
\hline $\begin{array}{l}\text { Angel Maquieira } \\
\text { Spain }\end{array}$ & $\begin{array}{l}\text { Kenneth Noll } \\
\text { USA }\end{array}$ & $\begin{array}{l}\text { Suresh Poda } \\
\text { USA }\end{array}$ \\
\hline $\begin{array}{l}\text { Eric Marechal } \\
\text { France }\end{array}$ & $\begin{array}{l}\text { Mohd Nor Norazmi } \\
\text { Malaysia }\end{array}$ & $\begin{array}{l}\text { Haryoung Poo } \\
\text { South Korea }\end{array}$ \\
\hline $\begin{array}{l}\text { Alexandra P. Marques } \\
\text { Portugal }\end{array}$ & $\begin{array}{l}\text { Santosh Noronha } \\
\text { India }\end{array}$ & $\begin{array}{l}\text { Rachel Poretsky } \\
\text { USA }\end{array}$ \\
\hline $\begin{array}{l}\text { Seamus Martin } \\
\text { Ireland }\end{array}$ & $\begin{array}{l}\text { Cory Nykiforuk } \\
\text { Canada }\end{array}$ & $\begin{array}{l}\text { Thomas Proft } \\
\text { New Zealand }\end{array}$ \\
\hline
\end{tabular}




\author{
Holger Puchta \\ Germany \\ Francoise Quignard \\ France
}

Arthur F.J. Ram

Netherlands

Sana Raouche

France

Mohammad Javad Rasaee

Iran

Peter Reilly

USA

Craig Richael

USA

Maria Manuela Rigano

Italy

Ana Filipa Rodrigues

Portugal

Maria Elisa Rodrigues

Portugal

Gabriela Rodrigues

Portugal

Alessandra Rogato

Italy

Fernando Rojo

Spain

M. Blanca Roncero

Spain

German Rosano

Argentina

Daniele Rosellini

Italy

Joy Roy

India

Scott Rudge

USA

Edward Rybicki

South Africa

Markus Sack

Germany

Xavier Saelens

Belgium

Debendra K. Sahoo

India
Kulvinder Saini

Saudi Arabia

Todd Sandrin

USA

Vitor Santo

Portugal

Nandini Sarkar

India

Pooja Saxena

USA

Joachim Schiemann

Germany

Monica Schmidt

USA

Pierre Schneeberger

Switzerland

Stefan Scholz

Germany

Richard Scott

New Zealand

Laura Segatori

USA

Junichi Sekiguchi

Japan

Davide Seruggia

Spain

Ole-Morten Seternes

Norway

Jian Shi

USA

Zhongping Shi

China

Kazuyuki Shimizu

Japan

Noriaki Shimizu

Japan

Rakesh Shukla

India

Sudhir Singh

India

Vanga Siva Reddy

India

Tobias Sjöblom

Sweden
Christopher Mark Smales

UK

\author{
Allison Snow \\ USA
}

Ulrich Soltmann

Germany

Ruth Elena Soria-Guerra

Mexico

Irma Soria-Mercado

Mexico

Oliver Spadiut

Austria

Rajan Sriraman

Germany

Vibha Srivastava

USA

Ralf Steuer

Germany

Eva Stoger

Austria

Krisztina Szabadfi

Hungary

Rouzbeh Taghizadeh

USA

Fuyuhiko Tamanoi

USA

Laszlo Tamas

Hungary

Sheng-Ce Tao

China

Maureen Taylor

UK

Matthew David Templeton

New Zealand

Eckhard Thines

Germany

Yigang Tong

China

Jorg Tost

France

Pei-Lan Tsou

USA

Maobing Tu

USA 
Satyanarayana Tulasi

India

Kiminori Ushida

Japan

Geertje Van Keulen

UK

Tom Van Wezel

Netherlands

Claudio Varotto

Italy

David Vaudry

France

Phanindra Velisetty

USA

Joachim Venus

Germany

Erik Vijgenboom

Netherlands

Eva Vincze

Denmark

Hans Visser

Netherlands

Antonia Vlahou

Greece

Moritz Von Stosch

Portugal

Masaru Wada

Japan

Ronny Wahlström

Finland

Kerstin Walter

Germany

Guoshun Wang

USA

Jiangxin Wang

China
Hao Wang

China

Yimeng Wang

USA

Yihai Wang

USA

Yun Wang

USA

Heribert Warzecha

Germany

Tatsuo Watanabe

Japan

Grzegorz Wegrzyn

Poland

Ziming Weng

USA

Nick Wierckx

Germany

Dominic Wong

USA

Gavin Wright

UK

Jia Xie

USA

Danke Xu

China

Xingfu Xu

USA

Zhenbo Xu

China

Montarop Yamabhai

Thailand

Boxu Yan

USA

William Yang

USA
Shinichi Yano

Japan

Weijuan Yao

China

Jaeseung Yoon

South Korea

Jianlan You

USA

Jamey Young

USA

Xiaobo Yu

USA

Qing-Yin Zeng

China

Bo Zhang

USA

Feng Zhang

USA

Shijun Zhang

USA

Wenli Zhang

Germany

Wei Zhang

Canada

Yongxin Zhao

Canada

Liang Zhao

USA

Fanfan Zhou

Australia

Huihao Zhou

USA

Jonathan Zuckerman USA 